\title{
The process of oncology nurse practitioner patient navigation: a grounded theory approach, navigation tools
}

\begin{abstract}
Evolving research has shown that oncology nurse practitioner navigators (ONPNs) improve clinical outcomes. ${ }^{1-4}$ Models for quality assurance such as the Donabedian. ${ }^{5}$ model have stressed the critical linkage between the role that processes have in determining outcomes, and the challenges involved in determining cause and effects of these components. According to the Donabedian. ${ }^{5}$ construct of structure, process, and outcome, each organizational factor is influenced by the previous. A study was undertaken. ${ }^{6}$ to better define the process that ONPNs use in caring for cancer patients which answered the question what processes do ONP navigators use in caring for cancer patient? A major finding of this study was that program development was evident in all phases of the navigation, and evolved to incorporate a navigation system. The navigation system involved a placement of navigators in key positions that would facilitate timely care. The thrust to deliver high quality timely cancer supports the current trend in health care which is the moving away from fee for service to bundled payment reimbursement. The trend towards capitated and bundled payments results in payment for blocks of care; thus laying the financial responsibility on the care delivery system to assume care for the specified time period. The Oncology Care Model (OCM) is a new healthcare delivery model that was designed to provide cost effective quality care for chemotherapy patients. Services center upon the provision of services emphasizing care coordination, navigation, and national benchmark treatment guidelines for care (CMS, 2017).
\end{abstract}

Inherent in any navigation program is the need for tracking, an essential component of the navigation process. ${ }^{6}$ This study revealed the use of navigation tools as the means of directing care, and collecting data for clinical trends. Thus the aim of this manuscript is to: (a) define the aim, philosophical orientation, methods, select findings of the Process of Oncology Nurse Practitioner Patient Navigation: A Grounded Theory Approach pertaining to tracking tools, and (b) discuss the use of navigation tools used for tracking in view of the current literature.
Volume 8 Issue 7 - 2017

Frances Johnson

Department of Defense, Carl R Darnall Army Medical Center, Medical Oncology, USA

Correspondence: Frances Johnson, PhD, ANP-BC, AOCN, CNS, 295 I Marina Bay 130-5 18, League City Texas, USA, Tel 281300-2635, Email roseypumpkin@mail.com

Received: November 29, 2017 | Published: December 14, 2017

\section{Research question}

To gain further insight into the processes that ONP navigators use in cancer care, a qualitative descriptive study was undertaken to answer the following research question: What processes do ONP navigators use in caring for cancer patients?

\section{Philosophical orientation}

Constructivism was the philosophical orientation for the study. Data and analyses are created from shared experiences and relationships with participants and other sources of data. ${ }^{7}$

\section{Methods}

\section{Procedure for collection and treatment of data}

Grounded theory was chosen as the methodology. It has defined guidelines that link theory with practical application resulting in theoretical explanation. ${ }^{8}$

\section{Setting}

The settings for the study included telephone interviews in a location convenient to the participant in any area of the United States. For the most part, the researcher conducted the telephone interviews from her private study behind closed doors located in her place of residence in Houston, Texas.

\section{Participants}

The study participants were recruited from all areas of the United States. To be eligible for the study, the nurse practitioners were required to be working as navigators and meet the following criteria: 1) license to practice as an NP in their respective state; 2) certification to practice as an oncology nurse; 3 ) minimum of 5 years full time experience in oncology nursing; and 4) English speaking.

\section{Data collection}

The research protocol involved an interview script, and interviews were conducted until theoretical saturation occurred. Theoretical saturation is defined as "the point in analysis when all categories are well developed in terms of properties, dimensions, and variations. Further data gathering and analysis add little new to the conceptualization, though variations can always be discovered".

\section{Data collection procedures}

Data collection procedures were generated by the emerging theory and mutually agreed upon by student and committee chair. Data collection was in the form of interviews using an initial interview 
script via the telephone. As the theory emerged the interview questions were revised on an ongoing basis.

\section{Sample}

The sample consisted of $\mathrm{N}=20$ nurse practitioners from all areas of the United States.

\section{Data analysis}

In grounded theory, data collection and analysis occur simultaneously. ${ }^{9}$ The goal of data collection in accordance with Social Interaction Theory was to learn the meaning that the NP navigators ascribe and internalize from the situation, and to determine the social construct of navigation formulated through this social interaction. ${ }^{10}$ Basic analytic techniques for the major study that were used in this grounded theory included sampling, memoing, constant comparison, and coding. ${ }^{9}$ Coding techniques included line by line, open, axial, selective and theoretical coding. Line by line coding allowed the researcher to remain open about the data and its individual parts, defining the actions of the process, identifying the tacit assumptions, and comparing the data amongst the participants. ${ }^{7}$ This led to the development of categories and processes. ${ }^{7}$ NVivo software for windows was used to guide this process.

A large part of the data for this paper has been extracted from one of the questions from the interview guide which is: What does you hospital do to monitor the navigation process in respect to process improvement?

\section{Findings}

The navigator ONP navigation process is a process that is carried out synergistically within the context of the patient, facility, and community. ${ }^{6}$; therefore navigator tools were identified in this study that crossed each of these three levels of care. ${ }^{6}$

The overall goal of the ONP navigation process is to expedite care along the cancer continuum. ${ }^{6}$ The core category for the process was identified as staying connected to the patient and to the system (Johnson, 2016). Timely care was gauged by metrics. ${ }^{6}$ Navigation tools were tied closely with these metrics, facilitated their tracking, and were used in all phases of the navigation process. ${ }^{6}$ Tracking and tracking tools were the means by which the navigator facilitated staying connected to the patient and system. The information from the tools is compared to standards of care and serves as a guide for patient care and process improvement. ${ }^{6}$.

\section{Navigation tools}

\section{Patient tools}

Examples of patient tools included chart review templates, Gail Model Risk Assessment, lung nodule screening criteria, triage protocols, and Press Ganey Scores. Less formal tracking tools included spreadsheets, task point, note template, sticky notes, informal face sheets, chart reviews, Excel spreadsheets, PowerPoint tools, Care Coordination master schedule, Outlook alerts, and triage protocol. Navigators may create forms specific to their needs, for example. "We have an intake and referral sheet. It's a 2 page form. Even though the hospital has an electronic medical record we're still using paper form and paper charting because the electronic health record doesn't have a navigation piece to it, and we need to be able to track when to follow-up with patients and when the patient's surgery is and when to call them back... We need to be able to see at a glance what's happening."."

\section{Facility tools}

Examples included sophisticated computer systems, leadership meetings for program evaluation, process tools, and pamphlets describing the navigator role with contact information, QA initiatives, and multidisciplinary meetings for consensus opinions regarding treatment planning. Computer tracking systems were utilized frequently as a means of communication between the systems. Journey Forward (n.d.) was popular for use in survivorship. This is a free tool that assists oncology professionals to make tailored treatment plans. The $\mathrm{CoC}$ maintains that it meets the requirements identified by them and the IOM for important components of survivorship care (Journey Forward, n. d.). Human trackers included $\mathrm{RN}$ data specialists. Administrative tools for system analysis and goal formation included process maps, picture representation of program, and specific navigation guidelines such as the NCOBC navigation steps. One program used an NCCP flowchart to standardize the navigation process amongst the different navigators within the system. Computer programs included ARIA ${ }^{\circledR} .^{11-16}$ as well as homegrown tailored computer software programs. ${ }^{6}$ One hospital system utilized a homegrown tailored computer software program designed for their system. ${ }^{6}$

"I am entering every step of the way for them behind the scenes, so everything from their diagnosis, imaging, abnormal imaging, biopsy and continuation from there, has all been populated all along the way...".

\section{Community tools}

These included marketing tools, group meetings, and community resource binders. Several participants described coordination of state and facility programs for cancer control through the use of a shared data base. RN Data Specialists... who also work with the -let me see, the (National Database of Nursing Quality Indicators) NDNQI. ${ }^{6}$

\section{Discussion}

\section{Patient tools}

Chart reviews: Chart reviews and accompanying templates are an established clinical research practice that has been used for over nine decades. Obtaining data from health records is common in epidemiological investigations, quality improvement studies, education of professionals, and review of inpatient care. ${ }^{17}$ Chart review templates are useful as a means of guiding and formulating prospective inquiries. Methodological issues to avoid include: failure to articulate the area of research, to consider sampling a priori, to operationalize variables, to monitor and train data abstractors, to use standardized abstraction forms, to create an adequate procedural manual for data abstraction, to clearly develop inclusion and exclusion criteria, to address inter/intra-rater reliability, to perform a pilot test, and to address ethical considerations. ${ }^{18}$ Properly designed templates can be developed as part of the navigation process to refine data collection methods so that data is robust, and guide process improvement measures for evidenced base care Gearing \& Mian et al. ${ }^{17}$ cite steps to ensure a quality chart review. Additionally, automated methods can be put in place to accelerate chart review data extraction through the use of the electronic medical record. ${ }^{19}$

\section{Risk assessments}

Validated risk assessment models such as the Gail Model are useful in patient navigation programs for guiding care and are in counseling endeavors, so the provider can help guide the patient 
in shared decision making counseling sessions. The PanCan Lung Cancer Risk Predictive Model. ${ }^{20}$ is a validated tool for lung cancer. A systemic review performed by Louie, Seigneurin, Cathcart, \& Sasient. ${ }^{21}$ indicated that six models met study criteria for metaanalysis for predicting prostate cancer. These included: Prostaclass, Finne, Karakiewcz, Prostate Cancer Prevention Trial (PCPT), Chun, and the European Randomized Study of Screening for Prostate Cancer Risk Calculator (ERSPC RC3).

Family Healthware, an internet-based tool that collects family history for six common diseases, identified patients who were in need of more intensified cancer screening. A randomized controlled clinical trial in primary care, involving patients aged 35-65 years, looked at cancer screening adherence and consultation. Measurement took place at baseline and at 6-months follow-up in a sample of $\mathrm{N}=3283$ patients. The results showed that $34 \%$ were at strong or moderate risk of at least one of the cancers. It identified additional participants for whom earlier screening (colon cancer, 4.4\%; breast cancer, women ages: 35-39 years, 9\%) or genetic assessment (colon cancer, 2.5\%; breast cancer, $10 \%$; and ovarian cancer, $4 \%$ ) may be indicated. Fewer than $50 \%$ were utilizing risk based screening. Screening adherence improved for all risk categories and there was no difference between the intervention and control groups. ${ }^{22}$

\section{Triage protocols}

Triage protocols are important in cancer, especially for those that are undergoing cancer treatment, as implementation has been proven to reduce emergency room visits. ${ }^{23}$ Documentation of all calls between a patient and triage nurse is essential, and forms, and/or documentation in the chart are need to be in place which are available for the patient's physician and other members of the care team to review. ${ }^{24}$ The pan-Canadian Oncology Symptom Triage and Remote Support (COSTaRS) had developed 13 evidenced based protocols for cancer symptom triage which can be adapted by patients and nurses globally. ${ }^{25}$

\section{Patient satisfaction}

The most frequently cited indicator for patient satisfaction (PS) in this study were Press Ganey scores. ${ }^{26}$ This organization works with over 26,000 health care organizations and $60 \%$ of the United States hospitals to improve quality of care by measuring patient experiences and providing consultation for solutions that promote a safe, quality experience for patients across the continuum of care. ${ }^{27}$ Patient satisfaction is a primary goal of the patient navigator. According to the National Institute of Health (NIH) patient navigation is "... personal, one-on-one assistance throughout the continuum of cancer services..." (NIH, n.d., para. 3). This it is not only important that reliable and valid tools are used to gauge patient satisfaction; but that these tools accurately gauge this metric along the cancer continuum.

Pascal et al ${ }^{28}$ noted that there is no existing measurement tool for PS that covers the the whole gamut of cancer care. The Patient Satisfaction with Cancer-Related Care tool (PSCC) was developed. Principle Component Analysis (PCA) resulted in a one-dimensional measure accounting for $62 \%$ of the variance. Internal consistency was high ( $\alpha$ ranging from 0.95 to 0.96 ). Face validity, convergent validity and divergent validity were satisfactory as indicated by moderate correlations with subscales of the CASE-Cancer (all ps $<0.01$ ). There were non-significant correlations (all ps $>0.05$ ) with age, primary language, marital status, and scores on the Rapid Estimate of Adult Literacy in Medicine (REALM) Long Form.
Van Hezewijk, Ranke, et al. ${ }^{29}$ used the Dutch version of the Patient Satisfaction Questionnaire III (PSQ) (Hagedoorn et al. 2003) which has undergone vigorous reliability and validity testing. Factor analysis was used to determine that a variety of care settings and patient groups were found to have an impact on patient satisfaction scores. Response bias was noted to have an effect on scores, and the matchedpair method was recommended for detection of response bias. This tool was found to be an appropriate measurement tool for patient satisfaction.

\section{Microsoft outlook systems}

The use of the Excel spreadsheets were used by those in the study. There was mention of use of the Microsoft Access, which can move the data base to a higher level. University of Florida Health. ${ }^{30}$ has a useful handout for the beginner that is interested in learning Microsoft Access. Microsoft Lync was used in some instances to communicate throughout the institution. Emails/ alerts were commonly done through Microsoft Outlook.

\section{Facility Tools}

Meetings: The majority of the navigators took part in multidisciplinary meetings concerning the patients that they were caring for. According to Hahlweg, Didi, Kriston, Harter, Nestoriuc, \& Scholl. ${ }^{31}$ few studies measure the quality of information presented and the team processes that are present in multidisciplinary meetings, as this influences the quality of the decision making. A rating scale called the Multidisciplinary Tumor Board Metric for the Observation of Decision-Making (MDT-MODe) was used to assess the quality of the presented information and team processes using $\mathrm{N}=249$ cases in $\mathrm{N}=29$ MDTMs. Cancer-specific medical information was considered high, while psychosocial information information low quality. In $25 \%$ of the cases there were no decisions, and $10 \%$ more than one treatment recommendation was given. Higher levels of medical and treatment indecision was associated with the likelihood of more than one treatment recommendation. The authors concluded that the quality of information was associated with the recommendation. Uncertainty related to more than one recommendation. Time constraints were an issue. The recommendation was to restructure the meetings wherever improvements could be made. Wildes, O'Donovan, Colloca, \& Cheung. ${ }^{32}$ recommend the use of Comprehensive Geriatric Assessment as well as geriatrician in informing the oncologists regarding the patient treatment decision. The recommendation was that high priority should be considered for the integration of the geriatrician into the multidisciplinary tumor board.

Process tools and quality assurance initiatives: The overall goal of the ONP navigation process is to expedite care along the cancer continuum. ${ }^{6}$ The basic social process is staying connected to the patient and to the system. ${ }^{6}$ Therefore it is essential to take an inventory of the facility system issues that impede timely care and system connectivity. Workflow care gaps impeding streamlined care must be addressed. Process mapping is an essential tool that was cited in the study that can be used as a means of visualizing system problems, and assist employees to work collaboratively to develop a game plan. ${ }^{33,34}$ The six sigma. ${ }^{35}$ quality assurance programs have been found to be useful in patient navigation. ${ }^{36}$ Hassan $^{37}$ utilizes the six sigma approach to provide comprehensive application of business process management to health care facilities utilizing specific steps and action plan.

Marketing: Some of the facility marketing tools that were utilized in the study were business cards, letters announcing navigator services 
with contact information, information cards with the navigator's picture and function in areas that cancer patients frequented.

\section{Pictorial representation of the hospital navigation program.}

This was cited by one navigator as being a useful tool that assisted in visualizing the future projected growth of the navigation program, as well as the way in which the program interfaced with the outlying community.

Navigation computer software: The navigation software programs that were used by the ONP's in this study were: ${ }^{11-16}$ as well as homegrown tailored computer software programs were utilized. ${ }^{6}$ The trend for computerized navigation software is evolving. Some of the initiatives include the integration of national guidelines such as NCCN. ${ }^{38}$, smartphone patient/provider apps ${ }^{39}$ as well programs that generate organizational work-flow. ${ }^{40}$

RN data specialists (also incorporated under community tools): The use of dedicated RNs to collect statistics that monitor compliance with breast cancer guidelines that worked with the ONP navigator was cited in this study.

NCCP flowchart: This tool was used as a means of standardizing the navigation process amongst a group of ONP navigators with different specialities. This group used the Association of Community Cancer Centers navigation matrix. ${ }^{41}$

\section{Community Tools}

Marketing: Marketing was mentioned as a strategy that was used to integrate the hospital based ONP with the community. For example if the facility was outsourcing care such as radiation, or surgical services, the navigator would go into the community to meet the key contacts in the community. Community assessments were done, which unfolded high risk areas. The example was given of a case where outreach work was done to determine community interest in lung cancer, and smoking cessation groups. Community in-services were done which addressed community interest and need.

Focus groups: Community focus groups have been shown to be useful as a means of learning patient centered information that is later integrated into protocol for patient care. ${ }^{42}$ (Tseung, S., \& Chang, F. 2017). Focus groups in this study were used for patient evaluation of services, as well as for determining community interest related to outreach efforts for high risk cancers.

Cancer registry: There was mention of a survivorship nurse practitioner in this study that was integrating survivorship statistics with the tumor registry utilizing a computer data-base, so that comparisons could be made with state and national statistics.

Comprehensive loose-leaf booklet for patient education: Several of the ONP navigators in the study favored the use of a patient education spiral loose-leaf notebook that could be updated regularly displaying a comprehensive gamut of hospital and community providers, programs, as well as self-help information for managing treatment side-effects. ${ }^{43,44}$

\section{Conclusion}

This manuscript describes information obtained from the parent study: The Process of Oncology Nurse Practitioner Patient Navigation: A Grounded Theory Approach, and describes the navigation tools that were cited by the ONP's in the study for tracking patient care, an essential focus of the navigation process. These tools similar to the navigation process relate to patient care on the patient, facility, and community level. As this this is a qualitative study, suggestions for practice is not generalizable to the population at large. Further research is needed which validates the use of these tools in further studies. Suggestion for further research could include the formation of a questionnaire, rating the usefulness of these type of tools by navigators. Once the usefulness is validated, refinement of tool structure can be developed.

\section{Acknowledgements}

This author would like to thank the $\mathrm{N}=20$ ONP navigators for their participation in this interesting study, and their enormous contribution towards furthering nursing knowledge.

\section{Conflicts of interest}

The authors have no conflict of interest.

\section{Acknowledgements}

None.

\section{References}

1. Robles L, Slogoff M, Ladwig SE, et al. The addition of a nurse practitioner to an inpatient surgical team results in improved use of resources. Surgery. 2011;150(4):711-717.

2. Rosales AR, Byrne D, Burnham C, et al. Comprehensive survivorship care with cost and revenue analysis. Journal of Oncology Practice. 2014;10(2):E81-E85.

3. Johnson FM. Systematic review of nurse practitioner oncology navigation metrics. Clinical Journal of Oncology Nursing. 2015;19(3):308-313.

4. Terzo L, Fleming M, Yechoor A, et al. Reducing unplanned admissions focusing on hospital admissions and emergency department visits for patients with head and neck cancer during radiation therapy. Clinical Journal of Oncology Nursing. 2017;21(3): 363-369.

5. Donabedian A. Evaluating the quality of medical care. The Milbank Memorial Fund Quarterly. 1996;83(4):691-729.

6. Johnson FM. The process of oncology nurse practitioner patient navigation: A grounded theory approach. (Unpublished doctoral dissertation). Texas Woman's University, Texas, USA. 2016.

7. Charmaz K. Constructing grounded theory: A practical guide through qualitative analysis. Los Angeles, CA: SAGE Publications Inc, USA. 2012.

8. Maz J. Employing a grounded theory approach: Core characteristics. British Journal of Cardiac Nursing. 2013;8(9):453-458.

9. Corbin J, Strauss A. Basics of qualitative research ( $3^{\text {rd }}$ Edn.), Thousand Oaks, CA: Sage Publications, USA. 2008.

10. Blumer H. Symbolic interactionism: Perspective and method. University of California Press. USA. 1969.

11. ARIA®. 2017.

12. EQUICARE. 2017.

13. Software advise. 2017

14. Aspentech. 2017.

15. ACT (2017).

16. Cordata Healthcare Innovations. 2017. 
17. Gearing RE, Mian IA, Barber J, et al. A methodology for conducting retrospective chart review research in child and adolescent psychology. Journal of the Canadian Academy of child and Adolescent Psychiatry. 2006;15(3):126-134.

18. Vassar M, Holzmann M. The retrospective chart review: Important methodological considerations. J Educ Eval Health Prof. 2013;10(12).

19. Hu Z, Melton GB, Moeller ND, et al. Accelerating chart review using automated methods on electronic health record data for postoperative complications. AMIA Annu Symp Proc. 2016;1822-1831.

20. Winkler WMM, Riel SJ, et al. Predictive accuracy of the PanCan Lung Cancer Risk Prediction Model-External validation based on the CT from the Danish lung cancer screening trial. European Radiology. 2015;25(10):3093-3099.

21. Louie KS, Seigneurin A, Cathcart P, et al. Do prostate cancer risk models improve the predictive accuracy of PSA screening: A meta-analysis, Annals of Oncology. 2015;26(5):848-864.

22. Rubinstein WS, Acheson LS, Neill OSM, et al. Clinical utility of family history of cancer screening and referral in primary care: A report from the Family Healthware Impact Trial. Genet Med. 2011;13(11): 056-965.

23. Hunis B, Alencar AJ, Castrellon AB, et al. Making steps to decrease emergency room visits with cancer: Our experience after participating in the ASCO Quality Care Symposium Journal of Clinical Oncology. 2016;34(7):51-51.

24. Towle E. Telephone triage in today's oncology practice. J Oncol Pract. 2009;5(2):61.

25. Stacey D, Green E, Ballantyne B, et al. Implementation of symptom protocols for nurses providing telephone-based cancer symptom management: A Comparative case study. Worldviews Evid Based Nurs. 2016;13(6):420-431.

26. Press Ganey. 2016.

27. University of Utah Healthcare. 2016. About the Press Ganey survey.

28. Jean PP, Fiscella K, Freund KM, et al. Structural and reliability analysis of a patient satisfaction with cancer-related care measure: A multi-site patient navigation research program study. Cancer. 2011;117(4):854 861.

29. Hezewijk M, Ranke GM, Nes JG, et al. Patients' needs and preferences in routine follow-up for early breast cancer: An evaluation of the changing role of the nurse practitioner. European Journal of Surgical Oncology. 2011;37(9):765-773.
30. University of Florida Health. Microsoft Access 2010: Select queries and crosstabs. 2010.

31. Hahlweg P, Didi S, Kriston L, et al. Process quality of decision-making in multidisciplinary cancer team meetings: a structured observational study. BMC Cancer. 2017;17(1):772.

32. Wildes TM, Donovan A, Colloca GF, et al. Tumor boards in geriatric oncology. Age Ageing. 2017;14:1-3.

33. Sharp A, Mc Dermott. Workflow modeling: Tools for process improvement ( $2^{\text {nd }}$ edn.) Norwood MA: Artech House, USA. 2009

34. E-Com Canada Inc. How to map a business process. 2011.

35. Lamm, MH, Eckel S, Daniels R, et al. Using team principles to improve outpatient adult infusion clinic chemotherapy preparation turnaround times. American Journal of Health-system Pharmac. 2015;72(13):11381146.

36. Gavriloff C. The impact of lean six sigma methodology on patient scheduling. Nursing Ecconomics. 2017;(35)4:189-193.

37. Hassan M. An application of business process management to healthcare facilities. Health Care Manager. 2017;36(2):147-163.

38. Pennic J. NCCN Chemotherapy Orders to Integrate with Epic's HER. 2014.

39. Jibb LA, Stevens BJ, Nathan PC, et al. Implementation and preliminary effectiveness of a real-time pain management smartphone app for adolescents with cancer: A multicenter pilot clinical study. Pediatric Blood Cancer. 2017;64(10).

40. Nursenav oncology. 2015

41. Association of Community Cancer Centers. NCCCP navigation matrix 2011.

42. Tseung S, Chang F. Transitioning from hospitals to the community: Perspectives of rehabilitation patients with neurological disorders and their service providers. Disability and Rehabilitation. 2017;39(23):2420 2427.

43. Hezewijk M, Hille ET, Scholten AN, et al. Professionals' opinion on follow-up in breast cancer patients: Perceived purpose and influence of patients' risk factors. European Journal of Surgical Oncology. 2007;37(3):217-224.

44. National Cancer Institute (n. d.) Patient navigator. 